\title{
Predictors of Postprandial Hypoglycemia After Gastric Bypass Surgery: a Retrospective Case-Control Study
}

\author{
Elric Zweck ${ }^{1,2,3} \cdot$ Matthias Hepprich ${ }^{1,4}$ (I) Marc Y. Donath ${ }^{1}$
}

Received: 13 November 2020 / Revised: 31 January 2021 / Accepted: 8 February 2021 / Published online: 23 February 2021

(C) The Author(s) 2021

\begin{abstract}
Background Postprandial hypoglycemia after bariatric surgery is an exigent disorder, often impacting the quality of life. Distinguishing clinically relevant hypoglycemic episodes from symptoms of other origin can be challenging. Diagnosis is demanding and often requires an extensive testing such as prolonged glucose tolerance or mixed-meal test. Therefore, we investigated whether baseline parameters of patients after gastric bypass with suspected hypoglycemia can predict the diagnosis. Methods We analyzed data from 35 patients after gastric bypass with suspected postprandial hypoglycemia and performed a standardized mixed-meal test. Hypoglycemia was defined by the appearance of typical symptoms, low plasma glucose, and relief of symptoms following glucose administration. Parameters that differed in patients with and without hypoglycemia during MMT were identified and evaluated for predictive precision using receiver operating characteristic (ROC) areas under the curve (AUC). Results Out of 35 patients, 19 (54\%) developed symptomatic hypoglycemia as a result of exaggerated insulin and C-peptide release in response to the mixed-meal. Hypoglycemic patients exhibited lower glycosylated hemoglobin $\mathrm{A} 1 \mathrm{c}\left(\mathrm{Hb}_{1 \mathrm{c}}\right)$ and higher absolute and relative weight loss from pre-surgery to study date. $\mathrm{HbA} 1 \mathrm{c}$ and absolute weight loss alone could achieve acceptable AUCs in ROC analyses ( 0.76 and 0.72 , respectively) but a combined score of absolute weight loss divided by HbA1c $(0.78)$ achieved the best AUC.

Conclusions $\mathrm{HbAlc}$ and weight loss differed in patients with and without symptomatic hypoglycemia during mixed-meal test. These baseline parameters could be used for screening of postprandial hypoglycemia in patients after gastric bypass and may facilitate the selection of patients requiring further evaluation.
\end{abstract}

Keywords Postprandial hypoglycemia $\cdot$ Late-dumping $\cdot$ Bariatric surgery $\cdot$ Mixed-meal test $\cdot$ Complications

Elric Zweck and Matthias Hepprich contributed equally to this work and share first authorship.

Matthias Hepprich

matthias.hepprich@usb.ch

1 Clinic of Endocrinology, Metabolism and Diabetes, University Hospital Basel, Petersgraben 4, 4031 Basel, Switzerland

2 Institute for Clinical Diabetology, German Diabetes Center, Leibniz Center for Diabetes Research at Heinrich Heine University, Auf'm Hennekamp 65, 40225 Düsseldorf, Germany

3 Division of Cardiology, Pulmonology and Vascular Medicine, Medical Faculty, Heinrich Heine University Düsseldorf, Moorenstrasse 5, 40225 Düsseldorf, Germany

4 Clinic of Endocrinology and Metabolic Disorders, Cantonal Hospital Olten, Basler Strasse 150, 4600 Olten, Switzerland

\section{Introduction}

Bariatric surgery is an effective treatment modality for obesity [1-3] with confirmed long-term safety and overall benefit regarding weight loss, components of the metabolic syndrome, quality of life, and survival [4-6].

Nevertheless, up to a third of postbariatric patients report symptoms of postprandial hyperinsulinemic hypoglycemia [7], whereas some studies report even higher prevalence rates in standardized test settings $[8,9]$ and severe hypoglycemic episodes occurring in less than $12 \%$ of patients $[10,11]$. However, the exact prevalence remains unknown. Affected patients may experience neuroglycopenic and vegetative symptoms with different intensity typically within $3 \mathrm{~h}$ after carbohydrate intake [12]. Postbariatric hypoglycemia may lead to an impairment of quality of life and to increased food intake with subsequent weight regain [13]. 
Known risk factors for hypoglycemia in postbariatric patients are younger age, female gender, greater postoperative loss of weight, and pre-operative high insulin sensitivity [14-16]. The diagnosis of hypoglycemia is often demanding and requires fulfillment of Whipple's triad often established with provocation testing $[13,14,16-18]$. These cumbersome and cost-intense tests require constant observation of the patient for several hours by health care professionals. Therefore, they should ideally only be performed in patients with a high a priori chance of hypoglycemia [17]. Furthermore, the lack of a reliable and simple screening tool may partly explain underdiagnosis of hypoglycemia in postbariatric patients [12].

$\mathrm{We}$, therefore, investigated whether baseline parameters of postbariatric patients can predict the occurrence of symptomatic hypoglycemia during a mixed-meal test.

\section{Methods}

\section{Data Source and Study Population}

We evaluated all data of patients after bariatric surgery who underwent mixed-meal testing because of symptoms suspicious for postprandial hypoglycemia at the Clinic for Endocrinology, Diabetes and Metabolism of the University Hospital Basel between May 2017 and October 2019, where approximately 100 patients undergo bariatric surgery annually. Patients are regularly followed up after bariatric surgery in our center. Participating patients presented with history of hypoglycemic symptoms and were therefore screened for this condition at our institution. In some of these patients, the provocation tests were also used as screening for a clinical trial (clinicaltrials.gov NCT03200782). Patients that underwent a MMT but also had diabetes mellitus $(n=1)$, gastric sleeve surgery $(n=1)$, or nesidioblastosis $(n=1)$ were excluded from analyses. All patients gave written informed consent for the use of their data.

\section{Standardized Liquid Meal Test}

Following an overnight fast, patients ingested a liquid $300 \mathrm{ml}$ of mixed-meal drink containing $450 \mathrm{kcal}$ and $60 \mathrm{~g}$ of carbohydrates (Ensure ${ }^{\circledR}$ Plus). Patients had to rest in a $45^{\circ}$ upright position for the whole test. Heart rate and blood pressure were assessed at baseline. Every 30 min bedside glucose was measured, and venous blood samples were drawn to assess glucose, insulin, and C-peptide. Occurrence of hypoglycemic symptoms was monitored by checking for typical symptoms according to the Edinburgh Hypoglycemia scale and neurocognitive questions comprising repetitive questions for date of birth, current date, serial subtraction of seven-test, repeating words, and backward spelling of words. In patients presenting with symptomatic hypoglycemia during the test, $10 \mathrm{~g}$ of glucose were administered intravenously or orally depending on severity of the symptoms by a physician. If symptoms persisted, glucose was given repetitively until symptoms resolved and blood glucose normalized. The test was terminated after two consecutive measurements of blood glucose rising again after the initial postprandial drop or after 210 min without any hypoglycemic symptoms.

At baseline, insulin resistance was estimated using Homeostasis Model Assessment (HOMA and HOMA2) [19]. Several additional indices for insulin sensitivity and insulin secretion using the data assessed during the MMT were estimated: Whole-body Insulin Sensitivity Index, Oral Glucose Insulin Sensitivity, Predicted M-value, Insulin Secretion Index I, and Insulin Secretion Index II [20-24].

\section{Statistical Analyses}

Primary outcome of interest was the occurrence of symptomatic hypoglycemia during a standardized MMT, defined as low plasma glucose $(<3.4 \mathrm{mM})$ concurrent with typical symptoms, which can be relieved by glucose administration (Whipple's triad). Statistical analyses were conducted with SPSS ${ }^{\circledR}$ Statistics 25.0.0.2 (IBM®, Armonk, NY, USA) and Python 3.7.4 (Python Software Foundation, DE, USA) with publicly available software packages (pandas, NumPy, Matplotlib, scikit-learn, tableOne, statsmodels, SciPy). In figures, data are presented as means \pm standard error of the mean and those given in tables are presented as median with interquartile range. Groups were compared using Kruskal-Wallis test. Receiver operating characteristic (ROC) curves were calculated for all continuous variables in the dataset. Areas under the curves (AUCs) of these ROC curves were calculated to identify most predictive variables. Univariate ROC analysis was chosen over multivariate logistic regression due to (i) the sample size, which was adequately powered for an univariate ROC with an alpha of 0.05 and a beta of 0.2 , but not sufficient for a multivariate linear model, and (ii) due to the easy clinical applicability of ROC due to its intrinsic advantage of providing cutoffs for the examined predictors. Univariate logistic regression was additionally performed to provide odd ratios for all continuous variables. For the creation of the score, highly co-linear variables with a Spearman rank correlation coefficient $>0.6$ were excluded by removing the variable with the smaller AUC in the ROC curve. Statistical significance threshold was $p<0.05$.

\section{Results}

\section{Baseline Characteristics of Patients}

Thirty-five patients were included in the analyses, 19 patients (54\%) presented with hypoglycemia, whereas in 16 patients (46\%), the suspicion of hypoglycemia could not be confirmed by the MMT. Baseline characteristics of both groups are 
depicted in Table 1. Both groups exhibited comparable age, sex distribution, and BMI. All patients had undergone Rouxen-Y gastric bypass surgery. At baseline, the only significant differences between the groups were $\mathrm{HbA}_{1 \mathrm{c}}$ and absolute and relative weight loss as compared to the weight pre-surgery (Table 1).

\section{Glucose Metabolism During Mixed-Meal Test}

We compared plasma glucose, insulin, and C-peptide levels during the MMT (Fig. 1). Meal intake induced an exaggerated immediate stimulation in insulin and C-peptide leading to hypoglycemic levels after $120 \mathrm{~min}$ in patients with hypoglycemia compared to patients without hypoglycemia. Insulin resistance did not differ between groups, whereas insulin secretion assessed by HOMA-beta and insulin secretion indices was numerically increased, without reaching statistical difference (Table 2).

Table 1 Participants' characteristics for patients without symptomatic hypoglycemia and with hypoglycemia after gastric bypass surgery

\section{Prediction of Occurrence of Postprandial Hypoglycemia}

Based on our findings from univariate group comparisons and all continuous variables' AUCs from univariate ROC curves (Supplemental Table 1), we identified $\mathrm{HbA}_{1 \mathrm{c}}$ and absolute weight loss as the most predictive parameters for occurrence of postprandial hypoglycemia in our dataset. Both variables achieved acceptable performance in ROC analyses (Fig. 2) with optimal cutoffs for $\mathrm{HbA}_{1 \mathrm{c}}$ at $5.3 \%$ (sensitivity $56 \%$, specificity $84 \%$ ) and weight loss of $38.2 \mathrm{~kg}$ (sensitivity $58 \%$, specificity $88 \%$ ), respectively (Supplemental Table 2). However, a combined score of the two variables, calculated as the ratio of absolute weight loss (in $\mathrm{kg}$ ) to current $\mathrm{HbA}_{1 \mathrm{c}}$ (in \%), achieved the highest AUC of 0.784 , using $7.2(\mathrm{~kg} / \%)$ as a cutoff and achieving a sensitivity of $68 \%$ and a specificity of $94 \%$ at the local maximum.

\begin{tabular}{|c|c|c|c|c|c|}
\hline Variable & Unit & Non-hypoglycemia & Hypoglycemia & $p$ & Missing \\
\hline$n$ & & 16 & 19 & & \\
\hline Age & years & $40.8(37.5,47.8)$ & $42.9(35.4,51.0)$ & 0.947 & 0 \\
\hline Years since surgery & years & $4.5(2.9,6.4)$ & $3.9(1.7,5.5)$ & 0.175 & 0 \\
\hline Sex (female) & & $14(87.5)$ & $16(84.2)$ & 1.000 & 0 \\
\hline T2DM pre-surgery & & $2(14.3)$ & $2(12.5)$ & 1.000 & 5 \\
\hline T2DM current & & $0(0)$ & $0(0)$ & 1 & 0 \\
\hline Weight pre-surgery & $\mathrm{kg}$ & $109.5(103.8,121.5)$ & $116.2(107.5,129.5)$ & 0.296 & 0 \\
\hline Weight current & $\mathrm{kg}$ & $78.5(69.0,91.0)$ & $78.7(68.8,82.5)$ & 0.619 & 0 \\
\hline Absolute weight loss & $\mathrm{kg}$ & $29.0(25.0,35.2)$ & $39.0(31.6,53.5)$ & 0.024 & 0 \\
\hline Relative weight loss & $\%$ & $28.3(23.1,30.8)$ & $35.0(27.7,45.6)$ & 0.024 & 0 \\
\hline BMI pre-surgery & $\mathrm{kg} / \mathrm{m}^{2}$ & $39.4(38.1,42.6)$ & $43.4(39.8,45.6)$ & 0.132 & 0 \\
\hline BMI current & $\mathrm{kg} / \mathrm{m}^{2}$ & $28.3(25.8,31.8)$ & $28.2(24.7,30.3)$ & 0.436 & 0 \\
\hline Change in BMI & $\mathrm{kg} / \mathrm{m}^{2}$ & $10.9(9.7,12.8)$ & $14.5(10.9,19.5)$ & 0.028 & 0 \\
\hline Relative change in BMI & $\%$ & $28.3(23.1,30.8)$ & $35.0(27.7,45.6)$ & 0.024 & 0 \\
\hline Systolic blood pressure & $\mathrm{mmHg}$ & $119.0(115.5,131.5)$ & $109.0(100.0,117.0)$ & 0.060 & 10 \\
\hline Diastolic blood pressure & $\mathrm{mmHg}$ & $80.0(68.8,82.5)$ & $71.0(68.0,81.0)$ & 0.462 & 10 \\
\hline Heart rate & $\min ^{-1}$ & $74.5(66.0,81.2)$ & $72.0(68.0,80.0)$ & 0.723 & 10 \\
\hline Baseline glucose & $\mathrm{mmol} / \mathrm{l}$ & $4.7(4.4,4.8)$ & $4.5(4.5,4.7)$ & 0.485 & 12 \\
\hline Baseline insulin & $\mathrm{mU} / 1$ & $5.5(3.5,8.5)$ & $6.0(4.3,9.6)$ & 0.832 & 16 \\
\hline Baseline C-peptide & $\mathrm{pmol} / 1$ & $609.0(519.8,739.5)$ & $693.5(579.8,740.0)$ & 0.512 & 17 \\
\hline $\mathrm{HbA}_{1 \mathrm{c}}$ & $\%$ & $5.3(5.0,5.7)$ & $4.9(4.7,5.2)$ & 0.009 & 0 \\
\hline Hemoglobin & $\mathrm{g} / 1$ & $133.0(126.8,145.5)$ & $126.5(122.2,135.8)$ & 0.097 & 1 \\
\hline C-reactive protein & $\mathrm{mg} / \mathrm{l}$ & $0.6(0.4,2.0)$ & $0.7(0.3,1.6)$ & 0.637 & 1 \\
\hline Glomerular filtration rate & $\mathrm{ml} / \mathrm{min} / 1.7$ & $99.0(86.2,112.0)$ & $103.5(99.2,111.2)$ & 0.333 & 1 \\
\hline HOMA-IR & & $1.2(0.7,1.8)$ & $1.3(0.9,2.1)$ & 0.401 & 4 \\
\hline HOMA-beta & & $81.3(53.0,124.2)$ & $127.7(84.0,159.4)$ & 0.139 & 4 \\
\hline HOMA2-IR & & $1.3(1.2,1.5)$ & $1.4(1.1,1.5)$ & 0.808 & 4 \\
\hline HOMA2-beta & & $132.1(110.6,150.1)$ & $138.8(123.2,153.2)$ & 0.612 & 4 \\
\hline
\end{tabular}

p values were calculated with Kruskal-Wallis test or Fisher's test. $\mathrm{HbA}_{1 \mathrm{c}}$, glycosylated hemoglobin $\mathrm{A}_{1 \mathrm{c}}$; HOMA, Homeostasis Model Assessment; IR, insulin resistance; $\mathrm{PBH}$, postbariatric hypoglycemia; T2DM, type 2 diabetes mellitus 

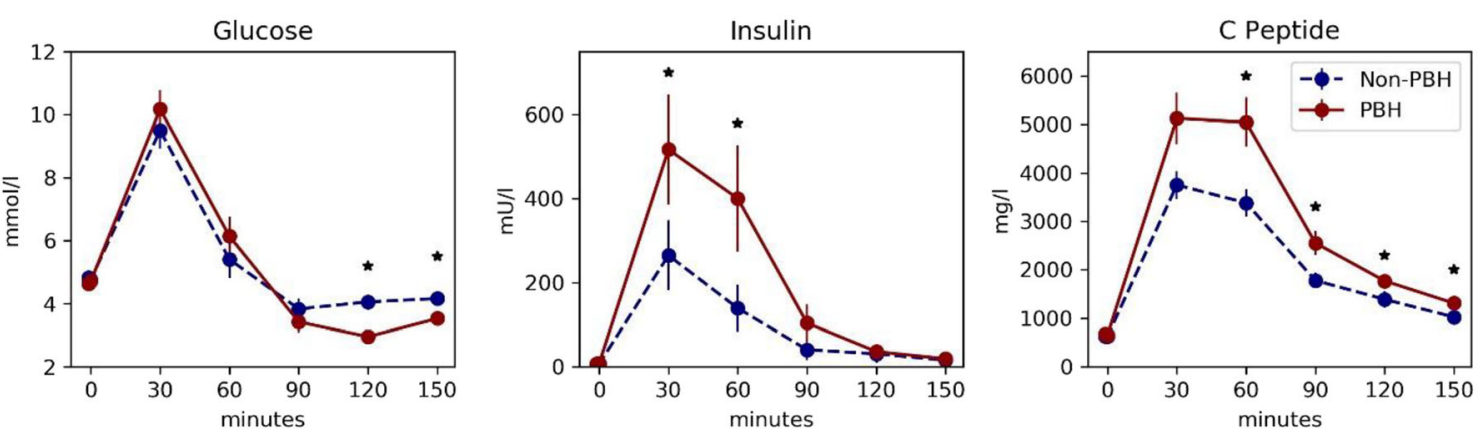

Fig. 1 Profiles for glucose, insulin, and C-peptide during a standardized mixed-meal test. Data are presented as mean \pm standard error of the mean. $n=29-36$ for each time point. $* p<0.05$ with Kruskal-Wallis test. Red

\section{Discussion}

It is presumed that the overall prevalence of hypoglycemia in postbariatric patients is underestimated [7,9]. One reason for this is the lack of standardized, practicable, and affordable screening tests. We demonstrate that in patients with suspected hypoglycemia, a score using $\mathrm{HbAlc}$ and the postoperative weight loss is able to identify patients at risk for symptomatic hypoglycemia during an MMT. We further depict baseline and in-test differences of patients with and without hypoglycemia. These observations might ease future screening for hypoglycemia in patients after gastric bypass surgery.

By performing an MMT with repeated measurement of glucose, insulin, and C-peptide, we could confirm the pathognomonic pattern of postbariatric hypoglycemia in our study cohort: in patients with hypoglycemia, insulin and Cpeptide increased drastically compared to patients without hypoglycemia, whereas glucose levels lowered markedly in the hypoglycemia group. With both groups starting from similar baseline values, these data also confirm the exclusively postprandial occurrence of hyperinsulinemia in the hypoglycemia group. These findings contrast previous studies reporting insignificant differences in postprandial glucose and insulin profiles between postbariatric patients with and those without postprandial symptoms [25, 26]. One possible explanation might be the larger number of patients in our cohort. solid line patients with symptomatic hypoglycemia and dashed blue line patients without symptomatic hypoglycemia after gastric bypass surgery

We used different indices for an estimation of insulin secretion capacity and insulin sensitivity, which did not indicate significant differences between patients with and without hypoglycemia regarding these parameters. This contrasts findings from a recent study by Raverdy et al. that suggested betacell function and insulin sensitivity might differentiate patients with and without hypoglycemia [14]. This difference to our study might result from the use of an oral glucose tolerance test in their study in contrast to a mixed-meal test in ours. Interpreting this is difficult due to the fact that none of these indices have been designed for postbariatric patients, hinting towards the need for more precise tools in these patients.

The outcome of interest for this study was the occurrence of late dumping, i.e., Whipple's triad, during a standardized MMT. This way, our study population's hypoglycemia status is well-characterized as opposed to large cohort or register studies, which define hypoglycemia by past diagnosis. In contrast to previous studies, age, sex, and fasting glucose did not distinguish patients with and without hypoglycemia in our study $[11,16,27]$. Higher preoperative insulin sensitivity may be another risk factor but was not assessed in this cohort [11]. In our trial cohort, lower $\mathrm{HbA}_{1 \mathrm{c}}$ and higher absolute weight loss clearly distinguished patients with and without PBH. Low $\mathrm{HbA}_{1 \mathrm{c}}$ could be explained by multiple episodes of hypoglycemia during the months before testing; however, it has not been reported in hypoglycemia patients yet. Greater weight loss in hypoglycemia patients has been reported in previous studies $[14,15,27,28]$ and might overstrain
Table 2 Indices of insulin secretion and insulin sensitivity during mixed-meal tests

\begin{tabular}{llll}
\hline Variable & Non-hypoglycemia & Hypoglycemia & $p$ \\
\hline$n$ & 12 & 18 & \\
Whole-body Insulin Sensitivity Index [22] & $103.5(91.1,120.6)$ & $72.8(43.1,115.6)$ & 0.099 \\
Oral Glucose Insulin Sensitivity [23] & $475.5(457.4,510.1)$ & $483.0(393.1,531.9)$ & 0.966 \\
Predicted M-value [24] & $2.4(2.1,2.4)$ & $2.6(2.2,2.7)$ & 0.225 \\
Insulin Secretion Index I [20] & $41.5(35.7,66.7)$ & $55.2(40.8,95.6)$ & 0.310 \\
Insulin Secretion Index II [21] & $20.5(15.5,31.4)$ & $32.3(20.1,48.8)$ & 0.189 \\
\hline
\end{tabular}

$p$ values were calculated with Kruskal-Wallis test 


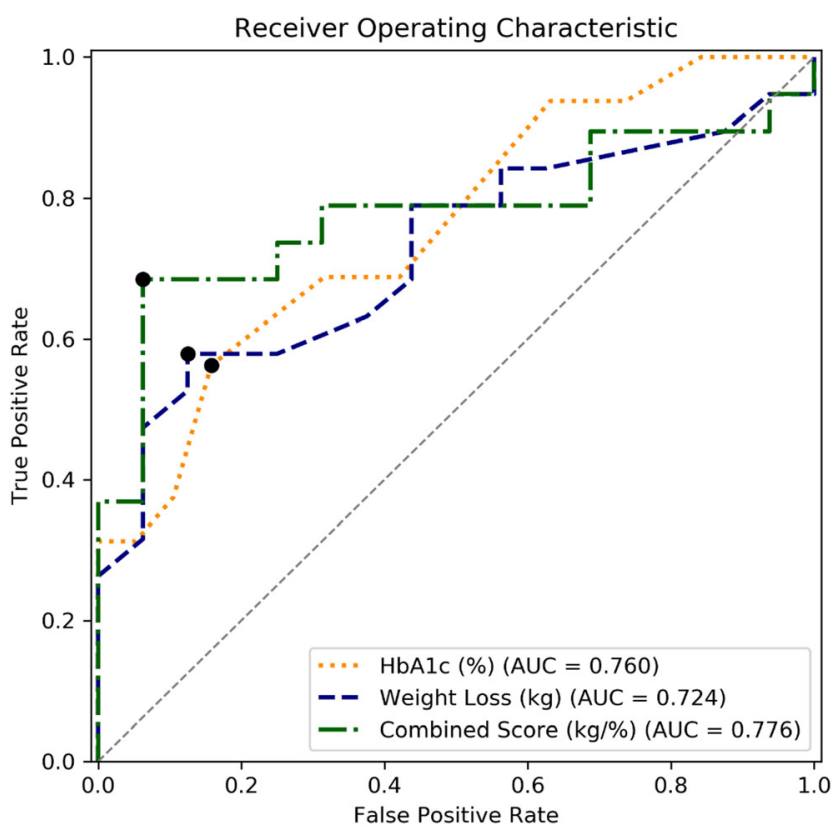

Fig. 2 Receiver operating characteristic curves. Curves are plotted with local optima, defined as the maximum of the true positive rate minus the false positive rate. The $\mathrm{HbA}_{1 \mathrm{c}}$ curve is displayed inversely for comparison. The combined score was calculated as the ratio of absolute postoperative weight loss $(\mathrm{kg})$ by $\mathrm{HbA}_{1 \mathrm{c}}(\%)$

metabolic adaptations after bariatric surgery, especially when occurring over a short period, leading to postprandial overexcretion of insulin. The mechanisms of this oversecretion appear to be multifactorial [29], and as recently discovered also mediated by interleukin $1-\beta[30]$.

At baseline, systolic blood pressure tended to be lower in patients with hypoglycemia. This might be driven by increased vagal activity or reflect lower concentrations of stress hormones preventing hypoglycemia in these patients. However, as using a single blood pressure measurement for screening is not recommendable due to possible fluctuation, we did not follow up on this in final ROC analyses.

While both HbAlc and weight loss achieved decent AUCs in the ROC analysis, combining the two into a score an even higher AUC of 0.776 was achieved. The score was calculated by dividing absolute weight loss by HbAlc. One of the major strengths of this score is its easy and wide applicability, as these parameters are usually assessed postsurgery in bariatric patients and $\mathrm{HbAlc}$ can be measured in most labs worldwide. By assessing medical history, hypoglycemia-related symptoms, and the score together in postbariatric patients, MMTs may be performed more targeted in the future.

\section{Limitations}

One possible limitation of this study is the use of a liquid mixed-meal test with fixed carbohydrate loads as opposed to body weight adapted loads. However, the current body weight was not predictive for the occurrence of symptomatic hypoglycemia and the median weight was similar in patients of both groups. These data do not suggest that baseline body weight had a relevant effect on the outcome of the MMT. However, the main limitation is the relatively small single center dataset, which limited the statistical analyses to be adequately performed, as well as the lack of a validation cohort. Therefore, our results and the proposed scoring system will require future studies to confirm their external validity, clinical applicability, and generalizability.

\section{Conclusion}

HbA1c, absolute, and relative postoperative weight loss differ at baseline in postbariatric patients with versus without symptomatic hypoglycemia during a standardized mixed-meal test. A proposed score of postoperative weight loss divided by HbAlc could predict the occurrence of PBH in a mixedmeal test. Future studies will provide insights into the generalizability of these results and the practicability of a wide use of our score as a screening tool in postbariatric patients.

Abbreviations AUC, Area under the curve; HbA1c, Glycosylated hemoglobin A1c; MMT, Mixed-meal test; ROC, Receiver operating characteristic; T2DM, Type 2 diabetes mellitus

Supplementary Information The online version contains supplementary material available at https://doi.org/10.1007/s11695-021-05277-1.

Funding Open Access funding provided by Universität Basel (Universitätsbibliothek Basel).

\section{Declarations}

Ethical Approval All procedures performed in studies involving human participants were in accordance with the ethical standards of the institutional and local ethics research committee and with the 1964 Helsinki declaration and its later amendments or comparable ethical standards.

Informed Consent Informed consent was obtained from all individual participants included in the study.

Conflict of Interest The authors declare that they have no conflict of interest.

Open Access This article is licensed under a Creative Commons Attribution 4.0 International License, which permits use, sharing, adaptation, distribution and reproduction in any medium or format, as long as you give appropriate credit to the original author(s) and the source, provide a link to the Creative Commons licence, and indicate if changes were made. The images or other third party material in this article are included in the article's Creative Commons licence, unless indicated otherwise in a credit line to the material. If material is not included in the article's Creative Commons licence and your intended use is not permitted by statutory regulation or exceeds the permitted use, you will need to obtain 
permission directly from the copyright holder. To view a copy of this licence, visit http://creativecommons.org/licenses/by/4.0/.

\section{References}

1. Angrisani L, Santonicola A, Iovino P, et al. Bariatric surgery worldwide 2013. Obes Surg. 2015;25(10):1822-32. https://doi.org/10. 1007/s11695-015-1657-z.

2. Welbourn R, Hollyman M, Kinsman R, et al. Bariatric surgery worldwide: baseline demographic description and one-year outcomes from the Fourth IFSO Global Registry Report 2018. Obes Surg. 2019;29(3):782-95. https://doi.org/10.1007/s11695-0183593-1.

3. Cheng J, Gao J, Shuai X, et al. The comprehensive summary of surgical versus non-surgical treatment for obesity: a systematic review and meta-analysis of randomized controlled trials. Oncotarget. 2016;7(26):39216-30. https://doi.org/10.18632/oncotarget.9581.

4. Sjostrom L, Lindroos AK, Peltonen M, et al. Lifestyle, diabetes, and cardiovascular risk factors 10 years after bariatric surgery. $\mathrm{N}$ Eng1 J Med. 2004;351(26):2683-93. https://doi.org/10.1056/ NEJMoa035622.

5. Bray GA, Frühbeck G, Ryan DH, et al. Management of obesity. The Lancet. 2016;387(10031):1947-56. https://doi.org/10.1016/ s0140-6736(16)00271-3.

6. Nguyen NT, Varela JE. Bariatric surgery for obesity and metabolic disorders: state of the art. Nat Rev Gastroenterol Hepatol. 2017;14(3):160-9. https://doi.org/10.1038/nrgastro.2016.170.

7. Gribsholt SB, Pedersen AM, Svensson E, et al. Prevalence of selfreported symptoms after gastric bypass surgery for obesity. JAMA Surg. 2016;151(6):504-11. https://doi.org/10.1001/jamasurg.2015. 5110.

8. Lazar LO, Sapojnikov S, Pines G, et al. Symptomatic and asymptomatic hypoglycemia post three different bariatric procedures: a common and severe complication. Endocr Pract. 2019; https://doi. org/10.4158/EP-2019-0185.

9. Capristo E, Panunzi S, De Gaetano A, et al. Incidence of Hypoglycemia after gastric bypass vs sleeve gastrectomy: a randomized trial. J Clin Endocrinol Metab. 2018;103(6):2136-46. https://doi.org/10.1210/jc.2017-01695.

10. Marsk R, Jonas E, Rasmussen F, et al. Nationwide cohort study of post-gastric bypass hypoglycaemia including 5,040 patients undergoing surgery for obesity in 1986-2006 in Sweden. Diabetologia. 2010;53(11):2307-11. https://doi.org/10.1007/s00125-010-17985 .

11. Lee CJ, Clark JM, Schweitzer M, et al. Prevalence of and risk factors for hypoglycemic symptoms after gastric bypass and sleeve gastrectomy. Obesity (Silver Spring). 2015;23(5):1079-84. https:// doi.org/10.1002/oby.21042

12. Patti M-E, Goldfine AB. The rollercoaster of post-bariatric hypoglycaemia. The Lancet Diabetes \& Endocrinology. 2016;4(2):94-6. https://doi.org/10.1016/s2213-8587(15)00460-x.

13. Malik S, Mitchell JE, Steffen K, et al. Recognition and management of hyperinsulinemic hypoglycemia after bariatric surgery. Obes Res Clin Pract. 2016;10(1):1-14. https://doi.org/10.1016/j.orcp.2015. 07.003 .

14. Raverdy V, Baud G, Pigeyre M, et al. Incidence and predictive factors of postprandial hyperinsulinemic hypoglycemia after Roux-en-Y gastric bypass: a five year longitudinal study. Ann Surg. 2016;264(5):878-85. https://doi.org/10.1097/SLA. 0000000000001915 .

15. Nielsen JB, Pedersen AM, Gribsholt SB, et al. Prevalence, severity, and predictors of symptoms of dumping and hypoglycemia after Roux-en-Y gastric bypass. Surg Obes Relat Dis. 2016;12(8):15628. https://doi.org/10.1016/j.soard.2016.04.017.
16. Belligoli A, Sanna M, Serra R, et al. Incidence and predictors of hypoglycemia 1 year after laparoscopic sleeve gastrectomy. Obes Surg. 2017;27(12):3179-86. https://doi.org/10.1007/s11695-0172742-2.

17. Emous M, Ubels FL, van Beek AP. Diagnostic tools for post-gastric bypass hypoglycaemia. Obes Rev. 2015;16(10):843-56. https:// doi.org/10.1111/obr.12307.

18. Scarpellini E, Arts J, Karamanolis G, Laurenius A, Siquini W, Suzuki H, et al. International consensus on the diagnosis and management of dumping syndrome. Nat Rev Endocrinol. 2020;16(8): 448-66.

19. Wallace TM, Levy JC, Matthews DR. Use and abuse of HOMA modeling. Diabetes Care. 2004;27(6):1487-95. https://doi.org/10. 2337/diacare.27.6.1487.

20. Phillips DI, Clark PM, Hales CN, et al. Understanding oral glucose tolerance: comparison of glucose or insulin measurements during the oral glucose tolerance test with specific measurements of insulin resistance and insulin secretion. Diabet Med. 1994;11(3):286-92. https://doi.org/10.1111/j.1464-5491.1994.tb00273.x.

21. Wareham NJ, Phillips DI, Byrne CD, et al. The 30 minute insulin incremental response in an oral glucose tolerance test as a measure of insulin secretion. Diabet Med. 1995;12(10):931. https://doi.org/ 10.1111/j.1464-5491.1995.tb00399.x.

22. Matsuda M, DeFronzo RA. Insulin sensitivity indices obtained from oral glucose tolerance testing: comparison with the euglycemic insulin clamp. Diabetes Care. 1999;22(9):1462-70. https://doi.org/10.2337/diacare.22.9.1462.

23. Mari A, Pacini G, Murphy E, et al. A model-based method for assessing insulin sensitivity from the oral glucose tolerance test. Diabetes Care. 2001;24(3):539-48. https://doi.org/10.2337/ diacare.24.3.539.

24. Tura A, Chemello G, Szendroedi J, et al. Prediction of clampderived insulin sensitivity from the oral glucose insulin sensitivity index. Diabetologia. 2018;61(5):1135-41. https://doi.org/10.1007/ s00125-018-4568-4.

25. Kim SH, Liu TC, Abbasi F, et al. Plasma glucose and insulin regulation is abnormal following gastric bypass surgery with or without neuroglycopenia. Obes Surg. 2009;19(11):1550-6. https://doi. org/10.1007/s11695-009-9893-8.

26. Laurenius A, Werling M, Le Roux CW, et al. More symptoms but similar blood glucose curve after oral carbohydrate provocation in patients with a history of hypoglycemia-like symptoms compared to asymptomatic patients after Roux-en-Y gastric bypass. Surg Obes Relat Dis. 2014;10(6):1047-54. https://doi.org/10.1016/j. soard.2014.04.007.

27. Brix JM, Kopp HP, Hollerl F, et al. Frequency of hypoglycaemia after different bariatric surgical procedures. Obes Facts. 2019;12(4): 397-406. https://doi.org/10.1159/000493735.

28. Lee CJ, Wood GC, Lazo M, et al. Risk of post-gastric bypass surgery hypoglycemia in nondiabetic individuals: a single center experience. Obesity (Silver Spring). 2016;24(6):1342-8. https:// doi.org/10.1002/oby.21479.

29. Salehi M, Vella A, McLaughlin T, et al. Hypoglycemia after gastric bypass surgery: current concepts and controversies. J Clin Endocrinol Metab. 2018;103(8):2815-26. https://doi.org/10.1210/ jc.2018-00528.

30. Hepprich M, Wiedemann SJ, Schelker BL, et al. Postprandial Hypoglycemia in patients after gastric bypass surgery is mediated by glucose-induced IL-1beta. Cell Metab. 2020;31(4):699-709 e5. https://doi.org/10.1016/j.cmet.2020.02.013.

Publisher's Note Springer Nature remains neutral with regard to jurisdictional claims in published maps and institutional affiliations. 